\title{
The potential of \\ bacteriocin-producing probiotics and associated caveats
}

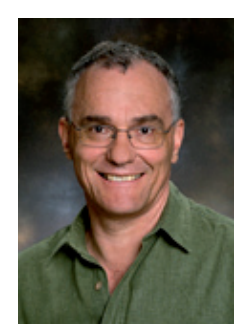

"...despite the fact that the production of two or

more bacteriocins by an E. colistrain is common, there are as yet poorly understood circumstances that seem to mitigate the advantages of multiple

bacteriocin production."

\section{David M Gordon}

Research School of Biology, Australian National University, Canberra, ACT 0200, Australia

" Tel.: +61261253552 = Fax: +61 261255573 m david.gordon@anu.edu.au

Most species of bacteria produce a diverse range of compounds that act as antimicrobial agents. These compounds include the broad-spectrum antibiotics produced by streptomycetes, lactic acid and hydrogen peroxide production by lactobacilli, and the production, by most bacteria, of protein antibiotics known as bacteriocins [1] Bacteriocins differ from other antimicrobial agents in that they tend to only be lethal to bacteria closely related to the species producing the toxin. The bacteriocins are abundant and diverse in both Gram-negative and Gram-positive bacteria, and there is now a wealth of theoretical and empirical evidence that shows bacteriocins are an important mediator of intra- and interspecific interactions and, consequently, a significant factor in maintaining microbial biodiversity [2] Bacteriocins are not only of interest to ecologists and evolutionary biologists, but are of increasing interest to workers in the fields of human and animal health, as well as to the food industry [2] . For example, the bacteriocin nisin, produced by Lactococcus lactis, has been used for many years as a food preservative to protect against contamination by Gram-positive organisms [3]. In addition, there are ongoing efforts to develop bacteriocins that might replace conventional antibiotics. For example, work is underway to modify the specificity of the pyocins produced by Pseudomonas aeruginosa [4]. Furthermore, bacteriocin production is thought to be a key trait for a successful probiotic strain and it is in the development of probiotics that bacteriocins appear to play their largest role [2]. There is a great deal of effort directed towards the development of probiotic strains that are effective against a variety of human GI tract pathogens, such as Listeria monocytogenes and Helicobacter pylori, as well as against pathogens of the oral cavity and vagina [2].
Not only are many potential probiotic strains, such as Nissle 1917, members of the species Escherichia coli, but much of our understanding of bacteriocins has also come from work with this species. E. coli is known to produce two diverse types of bacteriocins: colicins and microcins [3]. Colicin proteins range in size from 25 to $80 \mathrm{kDa}$, whilst microcin proteins are all less than $10 \mathrm{kDa}$. At a minimum, the bacteriocin gene cluster consists of a gene that encodes the toxin, and a constitutively expressed immunity gene whose product protects the cell from the bacteriocin. E. coli colicin gene clusters are plasmid encoded, whilst microcin gene clusters can be plasmid or chromosomally encoded. Many colicin determinants also encode a lysis protein. This protein ruptures the cell, releasing the colicin into the environment. If a lysis protein is not produced, the colicin is actively transported across the cell membrane into the external environment. Microcins are actively secreted from the cell. Once released, bacteriocin molecules bind to specific cell-surface receptors on target bacteria and are transported into the cell. Once the bacteriocin has entered the target cell it will kill the cell in one of a variety of ways, depending on the bacteriocin, such as by disrupting the cytoplasmic membrane integrity or by inhibiting protein synthesis. It is generally thought that a single bacteriocin molecule is sufficient to kill a sensitive cell.

Resistance to bacteriocins comes about through the loss or modification of the receptors on target bacteria used by the bacteriocin or in the pathway (TolA or TonB) used for the uptake of bacteriocin molecules into the target cell. Resistance to a wide variety of colicins is common in natural isolates of $E$. coli [5], and in vitro experiments suggest that while the cost of resistance can be quite high, these costs are

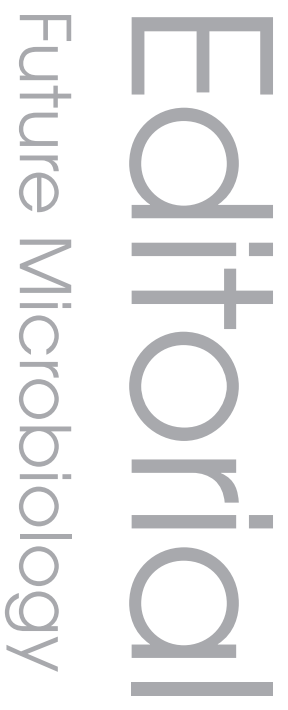

"Resistance to a wide
variety of colicins is
common in natural
isolates of E. coli, and
in vitro experiments
suggest that while the cost
of resistance can be quite
high, these costs are by no
means universal."

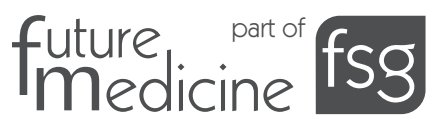


"...microcins may act to enhance a strain's ability to establish and maintain an infection in extra-intestinal body sites when in competition with other bacteria." by no means universal [6]. In theory, the evolution of bacteriocin resistance will be slowed if a strain produces two or more bacteriocins that each exploit a different receptor on target cells. This outcome results because the simultaneous occurrence of mutations in two different receptors is far less likely than a mutation in a single receptor gene. The production of multiple bacteriocins by a strain also confers the additional advantage of an expanded killing breadth. A strain that produces two different bacteriocins can kill cells that produce only one of the bacteriocins as well as being able to kill sensitive nonproducers. It appears that the majority of bacteriocin-producing $E$. coli strains produce two or more bacteriocins [7].

The production of more than one bacteriocin by a strain is, at least in theory, a desirable outcome that researchers often try to achieve when creating probiotic strains. Yet, despite the fact that the production of two or more bacteriocins by an $E$. coli strain is common, there are as yet poorly understood circumstances that seem to mitigate the advantages of multiple bacteriocin production. The coproduction of colicins $\mathrm{B}$ and $\mathrm{M}$ is common in E. coli isolated from native animals [8]. However, among isolates from humans and poultry, most strains that once encoded both colicins $\mathrm{B}$ and $\mathrm{M}$ have lost the ability to produce colicin B as well as their immunity to colicin B. This loss has apparently been driven by the gain of traits, such as iron uptake or serum survival, typically associated with plasmids that encode for the production of microcin $\mathrm{V}$ [9]. However, in most cases, the acquisition of microcin V-associated traits is not associated with the gain of microcin $\mathrm{V}$ production, just the loss of colicin B activity $[8,9]$. Another very common coassociation of bacteriocins in E. coli is between colicin Ia and microcin V [10]. However, as both the bacteriocins exploit the same (Cir) receptor, delaying the onset of resistance is an unlikely outcome of this co-association. Neither does it appear that the co-occurrence of these two bacteriocins results in an expanded killing breadth. In vitro experiments indicate that, although a strain that produces both colicin Ia and microcin $\mathrm{V}$ can invade a population of a strain that produces only colicin Ia, a strain that produces just colicin Ia can also invade a population dominated by a strain that produces both bacteriocins [Gordon D, Unpublished Data]. Consequently, while there appear to be advantages to multiple bacteriocin production in theory, in practice there appear to be other factors that can result in greater fitness benefits than those that arise through the production of multiple bacteriocins. Indeed, it appears that acquiring traits that enhance the ability of a strain to cause extraintestinal infection confers a greater advantage than multiple bacteriocin production.

Iron is an essential resource for almost all bacteria [11]. Bacteria can solve their iron requirements in several ways, and one of the most important is the production and secretion of high-affinity extracellular ferric chelators known as siderophores. The extracellular ferri-siderophore complexes bind to specific receptors on the outer membrane of the cell and are then transported into the cell via a number of steps [11]. Virtually all $E$. coli possess the siderophore receptors FhuE, FhuA, FecA, FepA, Cir and Fiu. $E$. coli may also possess the other iron-uptake systems, aerobactin, salmochelin and yersiniabactin, as well as the siderophore receptors Iha and IreA. However, the distribution of these systems among $E$. coli strains is highly variable.

There is an intimate link between microcins and iron. Many microcins are induced under conditions of iron limitation and many exploit receptors on sensitive cells that are involved in iron acquisition: Cir, FepA, FhuA, FyuA and IroN [12]. Furthermore, all strains encoding a microcin also possess one or more of the ironuptake systems aerobactin, salmochelin or yersiniabactin [7]. By contrast, significantly fewer $(60 \%)$ of nonmicrocin-producing strains encode one or more of these iron-uptake systems [7].

These observations are consistent with the hypothesis that microcins may mediate among-strain competition for iron. If true, then microcins may act to enhance a strain's ability to establish and maintain an infection in extra-intestinal body sites when in competition with other bacteria. Not only will microcinproducing strains be able to kill sensitive cells, but given that many microcins target one or more siderophore receptors, then the evolution of resistance to these microcins may well reduce the ability of the resistant strain to acquire iron. Although the resistant cell will not be killed by the microcin-producing strain it may well be outcompeted by the producer, as microcin producers encode multiple iron acquisition systems whilst many nonproducer strains do not. Furthermore, as siderophore production is also induced under conditions of iron limitation, resistant cells may have the additional burden of siderophore production.

This hypothesis assumes that multiclonal or multispecies extra-intestinal infections must be relatively common. Given that extra-intestinal 
infections arise as a consequence of contamination, there is little reason to suppose that the contaminating material harbors a monoculture of bacteria. There have been relatively few studies whose aim has been to determine the frequency of multigenotype extra-intestinal infections, especially during the early stages of an infection. However, the evidence indicates that multigenotype extra-intestinal infections are not infrequent. In one study, for example, multiple genotypes (two-five) were detected in $69 \%$ of E. coli-positive urine samples [13].

If microcins are mediating competition for iron among different genotypes attempting to establish an infection in an extra-intestinal body site, then microcin production could be thought of as a virulence trait. However, such a statement appears to be at odds with the experimental evidence. The presence of large conjugative plasmids encoding microcin $\mathrm{V}$ is known to be associated with $E$. coli virulence [14], but microcin $\mathrm{V}$ production itself is not thought to contribute to a strain's ability to cause disease [14]. Rather, other traits encoded by microcin $V$ plasmids, such as aerobactin and salmochelin, and the increased serum survival gene iss, have been implicated for the increased virulence of strains harboring microcin V plasmids [14]. However, these experimental studies are not incompatible with the idea that microcin production may be enhancing a strain's ability to compete for iron in mixed genotype infections. The experiments attempting to elucidate the traits on microcin V plasmids that contribute to virulence have involved single and not multiple genotype infections. Hence, interstrain competition for iron could not occur.

Much more experimental evidence is required in order to determine whether microcins mediate competition for iron. If true, then the inclusion of microcin production as a trait in probiotic bacteria (e.g., microcins $\mathrm{H} 47$ and $\mathrm{M}$ in Nissle 1917 [15]) may conceivably enhance the ability of the strain to cause disease if it succeeds in entering an extra-intestinal body site.

\section{Financial \& competing interests disclosure \\ The author has no relevant affiliations or financial involvement with any organization or entity with a financial interest in or financial conflict with the sub- ject matter or materials discussed in the manuscript. This includes employment, consultancies, honoraria, stock ownership or options, expert testimony, grants or patents received or pending, or royalties. \\ No writing assistance was utilized in the production of this manuscript.}

\section{Bibliography}

1. Riley MA, Wertz JE: Bacteriocins: evolution, ecology and application. Annu. Rev. Microbiol. 56, 117-137 (2002).

2. Gillor O, Etzion A, Riley MA: The dual role of bacteriocins as anti- and probiotics. Appl. Microbiol. Biotechnol. 81, 591-606 (2008).

3. Gillor O, Kirkup BC, Riley MA: Colicins and microcins: the next generation antimicrobials. Adv. Appl. Microbiol. 54, 129-146 (2004).

4. Williams SR, Gebhart D, Martin DW, Scholl D: Retargeting R-type pyocins to generate novel bactericidal protein complexes. Appl. Environ. Microbiol. 74, 3868-3876 (2008).

5. Riley MA, Gordon DM: A model of intraspecific microbial warfare. Trends Microbiol. 7, 129-133 (1999).

6. Feldgarden M, Riley MA: The phenotypic and fitness effects of colicin resistance in Escherichia coli K12. Evolution 53, 1019-1027 (1999).

7. Gordon DM, O'Brien CL: Bacteriocin diversity and the frequency of multiple bacteriocin production in Escherichia coli. Microbiology 152, 3239-3244 (2006).
8. Christensen J, Gordon DM: The evolution of colcin $\mathrm{M}$ and colicin B plasmids: the loss of B activity. Microbiology 155, 1645-1655 (2009).

9. Johnson TJ, Johnson SJ, Nolan LK: Complete DNA sequence of a ColBM plasmid from avian pathogenic Escherichia coli suggests that it evolved from closely related ColV virulence plasmids. J. Bacteriol. 188, 5975-5983 (2006).

10. Jeziorowski A, Gordon DM: Evolution of microcin $\mathrm{V}$ and colicin Ia plasmids in Escherichia coli. J. Bacteriol. 189, 7045-7052 (2007).

11. Andrews SC, Robinson AK, Rodriguez-Quinones F: Bacterial iron homeostasis. FEMS Microbiol. Rev. 27, 215-237 (2003).

12. Braun V, Patzer SI, Hantke K: Ton-dependent colicins and microcins: modular design and evolution. Biochimie 84, 365-380 (2002).

13. Schlager TA, Hendley JO, Bell AL, Whittam TS: Clonal diversity of Escherichia coli colonizing stools and urinary tracts of young girls. Infect. Immun. 70, 1225-1229 (2002).
14. Skyberg JA, Johnson TJ, Johnson JR, Clabots C, Logue CM, Nolan LK: Acquisition of avian pathogenic Escherichia coli plasmids by a commensal E. coli isolate enhances it abilities to kill chicken embryos, grow in human urine, and colonize the murine kidney. Infect. Immun. 74, 6287-6292 (2006).

15. Patzer SI, Baquero MR, Bravo D, Moreno F, Hantke K: The colicin G, H and $\mathrm{X}$ determinants encode microcins $\mathrm{M}$ and H47, which might utilize the catecholate siderophore receptors FepA, Cir, Fiu and IroN. Microbiology 149, 2557-2570 (2003).

\section{Affiliation}

- David M Gordon Research School of Biology, Australian National University, Canberra, ACT 0200, Australia Tel.: +61261253552

Fax: +61 261255573 david.gordon@anu.edu.au 